\title{
L'environnement dans le Pacifique Sud. Présentation
}

Jean Trichet et Isabelle Leblic

\section{(2) OpenEdition \\ 1 Journals}

\section{Édition électronique}

URL : http://journals.openedition.org/jso/4892

DOI : $10.4000 /$ jso.4892

ISSN : $1760-7256$

\section{Éditeur}

Société des océanistes

\section{Édition imprimée}

Date de publication : 15 décembre 2008

Pagination : 5-10

ISBN : 978-2-85430-012-3

ISSN : 0300-953x

Référence électronique

Jean Trichet et Isabelle Leblic, «L'environnement dans le Pacifique Sud. Présentation », Journal de la Société des Océanistes [En ligne], 126-127 | Année 2008, mis en ligne le 01 décembre 2008, consulté le 24 septembre 2020. URL : http://journals.openedition.org/jso/4892 ; DOI : https://doi.org/10.4000/jso. 4892 


\title{
L'environnement dans le Pacifique Sud Présentation
}

par

\author{
Jean TRICHET* et Isabelle LEBLIC**
}

Le Journal de la Société des Océanistes a souhaité examiner à nouveau dans ce volume 126127 (2008 - 1/2) la question de L'environnement dans le Pacifique Sud, question importante à plus d'un titre, qui justifie que nous y revenions régulièrement.

Ce numéro vient après deux autres volumes récents consacrés de près ou de loin à des thèmes proches ${ }^{1}$. Tout d'abord, en 2002, le JSO 114-115 qui était en Hommage à Jacques Barrau, grand inspirateur de l'ethnoécologie française (GuilleEscuret, 1997) et « personnalité incontournable pour la recherche océanienne » (Coiffier, 2002), qui nous a permis de revenir sur de nombreuses questions botaniques allant de la façon dont les Océaniens constituent leurs réserves alimentaires - en plantes sauvages ou cultivées - en passant par les techniques de domestication des plantes, sans oublier la relation avec l'animal mangeur de tubercules qu'est le porc. Ce numéro, très ethnologique, laissait aussi une grande place à l'implication des végétaux dans les relations sociales et spirituelles (Coiffier, 2002). Puis, plus près de nous, en 2005 , le $J S O$ 120-121 qui fut consacré à L'ethnoécologie en Océanie a présenté, à partir des communications de la table ronde De Ralph Bulmer à Peter Dwyer : pour une nouvelle approche ethnoécologi- que en Océanie organisée pour la Société des Océanistes par Florence Brunois et Christian Coiffier (au musée de l'Homme en avril 2003), auxquelles plusieurs papiers furent ajoutés, présenta diverses contributions en la matière. Par le passé, d'autres numéros thématiques avaient eu à voir, peu ou prou, avec la thématique de l'environnement. On peut citer par exemple le JSO 82-83 (1986) sur Les plantations dans le Pacifique Sud, ou encore, le 72-73 (1981) sur La pêche traditionnelle en Océanie, tous deux très orientés sur les populations et leurs activités sur l'environnement; enfin, le JSO 60 (1978): Noms de poissons marquisiens, mêlant spécialistes des sciences que l'on disait à une certaine époque « naturelles » avec divers autres plus humanistes.

Mais, aujourd'hui, notre dossier est nettement pluridisciplinaire et croise les apports tant des naturalistes que des spécialistes des sciences humaines et sociales (sHs). Nous renouons ainsi avec le caractère nettement multidisciplinaire de notre société, même si depuis quelques années, la dominante sHs avait pris le dessus. Ce volume rejoint ainsi les numéros thématiques plus «sciences de la vie » tels que les JSO 62 (1979): L'environnement de l'atoll de Takapoto-Tuamotu, ou 77 (1983): Récifs et lagons de Polynésie françaises.

1. En dehors des numéros spécifiquement thématiques, divers articles ont à voir avec les questions de l'environnement, sans pour cela l'avoir noté dans leurs mots-clés. Nous renvoyons les lecteurs aux index disponibles sur le site de la société des Océanistes (jusqu'en 1994, sur http://oceanistes.org, puis à partir de 2000, sur http://jso.revues.org; entre les deux, les index ne sont pas encore réalisés).

* Professeur des universités honoraire, jgtrichet@tele2.fr.

** CNRS LACITO, UMR 7I07, Villejuif, leblic@vjf.cnrs.fr. 


\section{Environnement : présentation d'un champ et quel- ques définitions}

L'environnement..., le sens de ce mot est vaste, puisqu'il concerne tant le climat d'une aire géographique donnée que ce qui détermine l'état d'âme d'un habitant de cette aire. Si le langage commun décrit surtout, sous ce mot d'environnement les composantes physiques (climatiques, thermiques, hydriques...) de domaines géographiques, marins ou continentaux, il l'applique aussi, de plus en plus, aux composantes psychologiques, historiques, sociales voire politiques intéressant les habitants de ces aires. Tous sont bien convaincus de l'importance de l'organisation des sociétés, de la psychologie des individus, de l'histoire des peuples et de la politique qu'ils choisissent ou qui leur est imposée, sur le climat, sensu lato, dans lequel ils vivent. Le mot « environnement » désigne donc, d'une manière générale, ce qui entoure un objet ou un être. Le mot «climat» a un sens à peine moins vaste, et d'ailleurs peu éloigné, puisqu'il désigne aussi bien les conditions physiques régnant à la surface des planètes que les conditions physiopsychologiques (un climat angoissant, un climat hostile) ressenties par un organisme.

Appliquer ces considérations à une aire géographique donnée, c'est donc en envisager, $a$ priori, toutes les composantes. Celles-ci sont de natures très diverses : climatiques - au sens habituel -, tout d'abord, car c'est du climat que procède toute vie. Montesquieu fut le premier à définir, magnifiquement, le climat comme "le plus grand magistère ». Et cela est bien vrai. $\mathrm{Au}$ demeurant, c'est essentiellement du climat atmosphérique au-dessus des continents qu'il s'agissait, les influences océaniques sur le climat étant alors peu connues. Puis, ce sont les composantes du sol, au sens le plus large du terme, que celles-ci soient aquatiques (domaines marins dans leurs variétés) ou terrestres, regroupant les roches et les sols - sensu stricto - dont les minéraux se sont développés à leurs dépens en contractant mariage avec la matière organique (humus) issue de la végétation. Ce sont, immédiatement après les sols, les composantes biologiques, végétales d'abord, animales ensuite. $\mathrm{Ce}$ sont ensuite les composantes anthropiques et humaines de l'environnement qui s'enracinent dans les composantes « physiques » précédentes. Les psychologies qui sont nées au contact de ces composantes physiques et les inventions successives qu'elles ont inspirées aux hommes au cours de leur histoire ne sont pas moins nombreuses que celles dans lesquelles elles ont puisé la source de leurs actions, de leurs inventions ou de leurs rêves : exploitation des sols, exploitation des ressources végétales, urbanisation, grands travaux, maîtrise des ressources en eau (irrigation), combustion immodérée de combustibles carbonés... L'homme laisse s'exprimer, dans ces actions, tous ses génies, bons et mauvais, et il y fait preuve tantôt de compétence ou d'incompétence, de prudence ou d'imprudence. Tous ces sujets relèvent donc bien de l'environnement humain. Mais s'ajoute à cette liste un sujet particulier. L'homme est aussi l'acteur de l'étude de son propre environnement, juge et partie. C'est un privilège qui le met, seul, à même de procéder à des choix utiles ou nuisibles à son environnement et nous avons choisi, pour cette raison, de donner leur place, dans ce numéro, au regard des chercheurs sur les programmes et les projets d'étude de l'environnement Pacifique qui s'y sont développés dans les années récentes et qui continuent à s'y développer.

L'environnement dans le domaine Pacifique est, comme tout vaste environnement, la somme et la coexistence de multiples environnements locaux. Et c'est dans ceux-ci qu'il convient de rechercher et d'explorer les diverses composantes évoquées ci-dessus. Il s'agit donc, tout d'abord et à nouveau, des caractéristiques physiques des environnements atmosphériques à la surface de $95 \%$ (99,5\% si l'on ne prend pas en compte l'Australie et la Nouvelle-Zélande) d'eaux marines et de $5 \%(0,5 \%$ sans l'Australie et la Nouvelle-Zélande) de terres, ces dernières toutes insulaires, toutes intertropicales, toutes situées dans l'hémisphère Sud (pour ce qui concerne les territoires et pays d'outre-mer possédant des liens avec la France, à l'exception de Clipperton, situé dans l'hémisphère Nord). C'est ensuite rechercher les propriétés des eaux et des sédiments marins ainsi que celles du sous-sol et des sols des îles émergées. C'est découvrir les flores et les faunes, tant marines que terrestres. C'est, enfin, pénétrer dans le chapitre complexe des environnements psychologiques, mentaux, historiques, sociologiques dont la somme constitue l'environnement humain du Pacifique. Et nous n'oublierons pas l'homme acteur de l'étude de son propre environnement.

\section{Paléoenvironnements et changements climatiques}

Avant de prendre connaissance de l'organisation entre ces différents thèmes des articles proposés dans ce numéro, il convient d'attirer l'attention sur la spécificité de l'un d'eux (Cabioch et al., pp. 25-39). Il traite en effet de l'évolution du paléoenvironnement (physique) 
de la Nouvelle-Calédonie au cours de la dernière déglaciation, c'est-à-dire depuis environ 18000 ans. Il rassemble donc des données essentiellement géologiques qui pourraient passer pour inutiles dans l'étude de l'environnement actuel. Se détourner de ces données serait commettre une grave faute de méthode car ce serait négliger une source d'informations essentielle sur l'environnement actuel. La grande leçon des études géologiques sur le Quaternaire est en effet que les variations des environnements à la surface de la terre ont une origine essentiellement climatique et qu'elles sont périodiques. C'est pour cette raison que les variations climatiques et biologiques observées, ou redoutées, dans les environnements actuels ne peuvent être comprises que si elles sont placées dans la continuité des variations subies pendant le Quaternaire récent. C'est pourquoi l'article de Guy Cabioch et al. a été rapproché de celui d'Anne Juillet-Leclerc (pp. 11-23) qui décrit, précisément, les marques laissées dans les coraux, pour l'essentiel fossiles, par ces variations climatiques prémonitoires de ce qui pourrait se produire jour dans notre environnement dans un avenir proche.

\section{Environnements physiques, atmosphériques et marins}

Plus avant dans ce dossier, les articles s'ordonnent de la manière suivante. La première série de contributions ouvrant cette section traite de l'environnement physique, atmosphérique et marin. L'article d'Anne Juillet-Leclerc (pp. 1123), tout d'abord, met en place les deux domaines fondamentaux - l'atmosphère et l'océan - au sein desquels et à travers lesquels se répartit l'énergie dont disposent les êtres vivants, dont les hommes, dans l'aire Pacifique. L'événement El Niño, spectaculaire dans l'océan Pacifique mais nullement limité à cet océan, y est tout particulièrement analysé. Suit l'article de Guy Cabioch et al. déjà évoqué (pp. 25-39) qui donne un éclairage paléoclimatique aux articles qui lui succèdent. Ainsi, Renaud Fichez et ses collaborateurs traitent dans leur article (pp. 41-58) des matériaux géologiques (sédiments côtiers) et biologiques (aquatiques) superficiels de la NouvelleCalédonie. Il introduit, à ce titre, les articles suivants traitant des relations entre l'homme et son environnement immédiat. Il développe en outre une perspective novatrice car les méthodes qui y sont exposées, présentées ici pour la première fois, ont été mises au point récemment, dans des programmes de lutte contre des pollutions, au sens large, dans l'environnement néo-calédonien.
Les trois articles suivants concernent l'environnement des récifs coralliens actuels, juste contribution à la connaissance de ces formations si spécifiques des océans intertropicaux et si réactives à leur environnement. Caroline Vieux et al. (pp. 59-66) décrivent les forces et contraintes notamment humaines - auxquelles sont soumises les lignes de rivage actuelles dans les îles de la Société (Polynésie française) et les déplacements qu'elles y induisent. Puis, Yannick Chancerelle et ses collaborateurs (pp. 67-74) décrivent une nouvelle et astucieuse méthode de relevés de la morphologie des récifs frangeants mise au point en Polynésie française, utilisant la photographie sous-marine pour de mesurer les modifications et les remaniements engendrés dans les environnements lagonaires, qu'ils soient d'origine humaine ou naturelle (tempêtes, cyclones...). Cette méthode, rapide et relativement précise sur des distances plus longues que celles techniquement observables jusqu'à présent, est donc très prometteuse. La contribution de Bernard Salvat et de Clive Wilkinson - "Initiative pour les Récifs coralliens (CRISP) » (pp. 75-93) - concerne la politique générale, à l'échelle du Pacifique, mise en œuvre pour promouvoir l'étude et la protection des récifs coralliens. En annexe, les auteurs nous présentent des listes de publications et communications des connaissances liées au programme CRISP.

\section{Environnements terrestres}

L'article de Bertrand Richer de Forges et de Michel Pascal, qui suit (pp. 95-111), est le premier consacré, dans ce numéro, à l'étude d'environnements terrestres, tous localisés en Nouvelle-Calédonie. Les mots-clés de son titre, nickel et biodiversité, indiquent à eux seuls l'objet des observations et des soucis des auteurs quant au respect et au maintien de la flore indigène d'un endémisme extraordinairement riche dans une île très touchée par les extractions minières. Article particulièrement d'actualité, quand on connaît l'ampleur des risques liés aux exploitations minières en cours de développement constant sur le Caillou (que ce soit dans le Sud avec Goro Nickel ou dans le Nord avec Koniambo Nickel sAs).

\section{Environnement : quelques histoires des relations de l'homme à son milieu}

Les thèmes des articles qui suivent diffèrent des précédents en ce qu'ils ne traitent pas seule- 
ment ou explicitement des objets naturels, mais se préoccupent aussi des manières avec lesquelles les hommes appréhendent leurs environnements, pour les exploiter, les dégrader, les agrader ou en organiser l'amélioration ou le sauvetage. L'histoire s'invite tout naturellement dans cette partie $\mathrm{du}$ volume puisque les générations héritent de celles qui les ont précédées les bienfaits ou les méfaits que les premières ont infligés à leurs paysages. Cette série d'articles commence avec Frédéric Angleviel (pp. 113-125) qui, en partant du voyage de circumnavigation de Magellan en 1521 et la période de découverte mutuelle qu'il a instaurée, notamment à partir de 1788 pour l'Océanie, retrace en détail l'histoire de l'anthropisation dans le Pacifique et les marques qu'elle a laissées dans l'environnement. À terme, la question de la gestion de l'environnement pour un développement durable des États insulaires du Pacifique Sud se pose. Marieke Blondet (pp. 127143) élargit le paysage de ce numéro jusqu'aux Samoa américaines où elle trouve des raisons historiques, psychologiques, voire cosmogoniques dans la réluctance dont font preuve les Samoans américains pour la protection de la nature de leurs îles. Elle se demande notamment si c'est le fait que les Samoans n'ont pas eu, autrefois ou récemment, à gérer des déchets non biodégradables qui fait qu'ils semblent mal se comporter avec leur nature aujourd'hui ou bien si c'est à cause d'un sentiment d'irresponsabilité qu'ils auraient vis-à-vis de ces espaces, sentiment ancré dans d'anciennes croyances cosmogoniques. Les effets de la colonisation américaine ne semblent pas à sous-estimer sur ce sujet, selon cette étude. Elsa Faugère, quant à elle (pp. 153166), argumente en faveur d'une nécessaire modernisation des approches dont doivent faire preuve tant les Occidentaux que les Insulaires du Pacifique, pour appréhender les objets naturels, par exemple par l'adoption d'un vocabulaire « moderne » pour décrire et nommer les espèces ou de traiter les grands nombres de données par des méthodes informatiques. Une telle " modernisation » doit s'appliquer aussi à tous les gestes politiques accompagnant l'établissement de projets ou d'établissements de recherche. Elsa Faugère, tout comme Marine Robillard (voir ci-dessous), deux ethnologues l'une et l'autre, s'intéressent ainsi aux questions de nomenclature ou de classification, plus sous l'angle des représentations autochtones que de celles des naturalistes. C'est là un point central des ethnosciences et il n'est pas sans intérêt d'en croiser la vision avec celle des scientifiques de la vie. L'objet de l'article de Guy Agniel (pp. 145-151), plus juridique, est dans la continuité, d'une part, de celui, historique, de Frédéric Angleviel et, d'autre part, du précédent, en traitant de l'ensemble des programmes mis en œuvre afin d'organiser la protection de certains environnements dans le Pacifique.

\section{Santo 2006 : une expédition naturaliste au $\mathrm{XXI}^{\mathrm{e}}$ siècle}

Dans cette partie, l'ensemble des articles, relatifs à des problématiques environnementales et humaines, fournit une illustration pratique des propositions, des déclarations ou des engagements discutés et proposés dans les articles précédents. Ces engagements ont été mis à l'épreuve des hommes et des faits dans la grande expédition naturaliste réalisée sur l'île de Santo, au Vanuatu, en 2006. Trop proche sans doute du retour de cette expédition, on pourra reprocher à notre publication de ne pas dégager suffisamment de conclusions concrètes, d'inventaires d'espèces découvertes, etc. Mais cela n'affaiblit pas, à nos yeux, la nécessité de parler de cette aventure dans notre journal, puisqu'elle constitue une première sur l'Océanie. Dans leur contribution liminaire (pp. 167-185), Philippe Bouchet, Hervé Le Guyader et Olivier Pascal, les représentants des trois organismes à l'origine de cette expédition (le Muséum national d'histoire naturelle, l'Institut de recherche pour le développement et l'oNG PRO-natura internantional), présentent le renouveau des explorations naturalistes des îles du Pacifique, retraçant l'histoire de ces expéditions depuis Cook, pour exposer l'origine de ce projet comme son déroulement sur place et introduire les autres articles qui constituent ce dossier Santo 2006. C'est ainsi que Michel Pascal et al. (pp. 187-193) étudient la place et la signification des espèces allochtones invasives dans le module appelé "friches et aliens ». Il n'est pas inutile de préciser sur ce point qu'il n'y a pas forcément de spécificités océaniennes concernant les invasions biologiques et leurs conséquences car celles-ci sont mondiales ( $c f$. Steadman, 2006). Elsa Faugère (pp. 195-205), associée à cette expédition comme ethnologue prenant pour sujet l'étude des biologistes occidentaux engagés dans celle-ci, expose ensuite la vision d'une observatrice non naturaliste sur l'organisation et sur le déroulement de l'opération Santo 2006. Elle y a été associée pour traiter de questions de sociologie de la recherche, celles des liens entre argent privé et science ou entre science et politique, auxquelles tout chercheur biologiste est confronté dans le contexte international à la suite de la signature de la 
convention sur la diversité biologique, ou encore celles concernant l'acquisition des savoirs scientifiques sur la biodiversité biologique. Cet article pose aussi la question du rôle d'une ethnologue en la matière censée aller au-delà de celui d'un simple porte-parole. Cette vision est à rapprocher de celle de Fabienne Tzérikiantz (pp. 207220) qui est beaucoup plus critique sur cette expédition, sa contribution faisant entrer de plain pied le lecteur dans cette opération socioscientifique. Spécialiste de la côte ouest de Santo où elle poursuit des enquêtes ethnologiques depuis 1996, elle pose plusieurs questions éthiques sur l'implication des partenaires locaux. Enfin, Marine Robillard (pp. 221-229) s'intéresse à la mise en perspective du regard occidental (celui des scientifiques de l'expédition) avec celui des habitants de Santo quant à la biodiversité. Elle insiste sur les points communs ou divergents entre les échelles de perception et les manières particulières de nommer et de classer les êtres vivants pour les uns et les autres.

\section{Quelques compléments hors dossier}

À la suite de ce dossier "spécial environnement» et «Santo 2006 », nous présentons comme à notre habitude quelques articles hors dossier. Certains trouvent leur place logique dans ce numéro sur l'environnement, tant les questions qui y sont évoquées sont proches des préoccupations recoupées ci-dessus. Cécile Gaspar et Tamatoa Bambridge (pp. 231-245), à propos des dynamiques territoriales liées à la mise en place d'Aires marines protégées dans le lagon de Moorea en Polynésie française, donnent un autre exemple de regards croisés, vétérinaire d'un côté et géographique de l'autre. Les auteurs présentent avec pédagogie les coutumes polynésiennes sur la gestion terre-mer et y replacent cette grande évolution, dans les coutumes et les mentalités, qui accompagna l'ouverture de la frontière terre-lagon. C'est une affaire qui a sans doute beaucoup intéressé l'environnement humain à l'époque et qui a donc toute sa place dans notre numéro. En outre, cet article montre bien toute la difficulté qu'il y a à se référer à des structures et des systèmes de pensées, notamment dans le domaine de l'environnement, dans des contextes culturels autres que celui qui a produit ces mêmes structures... et toute la difficulté et les logiques paradoxales, voire incompréhensibles, qui s'affrontent... Gilbert David (pp. 247-269) revient sur la vulnérabilité de la pêche côtière à l'instabilité du milieu naturel en Océanie, que ce soit à cause des aléas de la météorologie (qui affectent la fréquence et la durée des sorties de pêche) qu'à cause des facteurs biotiques et abiotiques qui régissent la présence du poisson et son abondance sur les lieux de capture. Cet auteur met l'accent sur les contraintes qu'impose l'environnement à l'acte de pêcher et sur les stratégies adaptatives que les pêcheur mettent en œuvre pour les minimiser, voire s'en affranchir. Mais ceux-ci sont euxmêmes acteurs de la transformation de leur environnement. Par leur prélèvement sur les stocks halieutiques, ils en ont modifié la dynamique démographique et se trouvent confrontés à un nouveau défi : comment réguler l'effort de pêche de manière à ce que leur activité puisse perdurer? La gestion territoriale et la création d'Aires marines protégées (AMP) apportent des solutions originales en ce domaine. En cela, l'article de Gilbert David complète bien celui de Cécile Gaspar et de Tamatoa Bambridge. Enfin, Pamela Swadling et al., dans leur article sur la préhistoire de l'agriculture en Nouvelle-Guinée, abordent deux questions encore sans réponse pour la préhistoire de cette île et de ses environs, savoir si l'agriculture était présente également dans les basses terres et dans l'archipel Bismarck à l'Holocène moyen et si cette présence a ou n'a pas été influencée par l'existence de relations avec la région des hautes terres.Les auteurs étudient l'utilisation des mortiers et des pilons des Enga comme marqueurs et proposent des réflexions novatrices pour les îles de l'archipel Bismark (pp. 271-292). Le dernier article présenté, celui de Bruno Saura (pp. 293-309) présente les manuscrits autochtones polynésiens du $\mathrm{XIX}^{\mathrm{e}}$ siècle dits puta tupuna en revenant plus spécifiquement sur ceux des îles Australes. Suivent dans les Miscellanées deux dossiers d'actualité : le premier, sur les bambous gravés kanak, en liaison avec l'exposition Bambous kanak. Une passion de Marguerite Lobsiger-Dellenbach présentée en début d'année au Musée ethnographique de Genève (MEG) de Genève et le catalogue qui l'accompagne (avec les contributions d'Isabelle Leblic [pp. 311-317], de Roger Boulay [pp. 318-319] et de Christian Coiffier [pp. 320325]) et, le second, sur l'exposition au musée du quai Branly L'aristocrate et ses cannibales. Le voyage en Océanie du comte Festetics de Tolna, 1893-1896 (pp. 327-334) par Gilles Bounoure (voir aussi son compte rendu d'un autre ouvrage sur cette exposition dans ce même numéro). Suivent les rubriques habituelles, les comptes rendus de lectures et les actualités de la société. 


\section{Conclusion}

Le champ des thèmes couverts dans ce numéro est vaste, allant de celui de la répartition des pressions atmosphériques au-dessus de la zone équatoriale à celui de l'harmonisation et de la gestion des programmes nécessaires pour protéger l'environnement, en passant par des techniques de mesure de la vitesse de courants. Mais il est un constat au-devant duquel il vaut mieux se porter soi-même, c'est celui de l'ambition du titre de ce double numéro. Traiter de «L'environnement du Pacifique » est une entreprise à la mesure même de cette aire géographique, donc considérable. À l'évidence, des réflexions et des données qui auraient été nécessaires pour une telle entreprise sont regrettablement absentes de la table des matières de ce cahier. Il en vient tant à l'esprit qui sont autant de pistes à suivre pour de futurs articles! Ce sont, par exemple, des considérations sur les problèmes de santé dans les îles, sur les problèmes relatifs aux sols et à l'agriculture, à l'hydrologie 2 , à la pollution littorale, à l'avenir de la pêche... Il en est même, peut-être, de plus urgents ou de plus graves: comment en est-on arrivé au décapage quasitotal de l'île de $\mathrm{Nauru}^{3}$, victime du fait que le phosphate qu'elle renfermait était d'origine lagonaire et qu'il en occupait, par conséquent, la majeure partie de la zone centrale? Que prévoir pour les atolls les plus bas, menacés par la montée du niveau marin sous l'influence de la fonte des glaciers d'inlandsis? Que prévoir aussi contre l'accumulation d'énergie au droit des zones équatoriales, associée à l'augmentation de la température atmosphérique mondiale et qui peut entraîner des phénomènes climatiques violents? Comment décontaminer aussi rigoureusement que possible les îles ayant servi de sites pour les essais nucléaires aériens des États-Unis, du Royaume-Uni et de la France, tout en restant attentifs à d'éventuelles manifestations des composés radioactifs profonds engendrés lors des tirs souterrains? Il faut protéger sans tarder les atolls fermés (Niau en Polynésie ; Clipperton...) ou partiellement fermés (Anaa en Polynésie...) et donc confinés du point de vue chimique, car le sédiment que contient leur lagon est fortement réducteur et concentre, à ce titre, nombre d'éléments polluants sinon nocifs, métaux lourds, hydrocarbures, pesticides ${ }^{4} .$.

La liste des agressions susceptibles de frapper telle ou telle partie de l'aire Pacifique, à commencer par de grands volumes de ses eaux marines elles-mêmes, est longue. L'immensité de son étendue maritime et la faible surface de ses terres émergées lui confèrent une spécificité remarquable, aux côtés des autres continents et des autres océans. La vie végétale et animale, puis la vie humaine, se sont remarquablement adaptées à ces conditions. Étudier ces territoires, c'est d'abord admirer ces adaptations. C'est aussi échanger et partager les savoirs, d'une région à une autre, faire connaître des moyens de vie, voire de survie, que nous ont appris, avec douceur ou brutalement, tous les environnements à travers le monde.

\section{RÉFÉRENCES BIBLIOGRAPHIQUES CITÉES}

CoIfFIER Christian, 2002. Avant-propos, Journal de la Société des Océanistes 114-115: Hommage à Jacques Barrau, pp. 5-8.

ELLIS Albert F., 1935. Ocean Island and Nauru: Their Story, Sydney, Angus and Robertson Limited, $319 \mathrm{p}$.

—, 1936. Adventuring in Coral Seas, Sydney, Angus and Robertson Limited, 318 p.

GuILlE-Escuret Georges, 1997. Jacques Barreau : un naturaliste au milieu des hommes (1925-1997, Journal de la Société des Océanistes 105, pp. 209-211.

Steadman D.W., 2006. Extinction and Biogeography of Tropical Pacific Birds, Chicago, Chicago University Press, 594 p.

2. Ce volume devait contenir un article sur l'hydrogéologie du Vanuatu rédigé par Michel Lepiller, maitre de conférences à l'université d'Orléans. Michel Lepiller nous a, hélas, quittés, à la suite d'un accident de la route. Nous rappelons ici sa mémoire avec émotion. Le lecteur intéressé par cette thématique pourra se référer à un article récent paru dans la revue Géoscience (Genthon et al., 2008) consacré à l'étude hydrogéologique de l'île de Lifou (île Loyauté, Nouvelle-Calédonie), étude à laquelle Michel a contribué.

3. Pour plus d'information voir la « confession » publiée en Australie dans les années trente du découvreur et exploiteur du phosphate de Nauru... (Ellis, 1935, 1936).

4. Cette question se pose d'ailleurs à un niveau mondial ! Qui n'a pas entendu parler sous toutes les latitudes de l'éradication des abeilles, notamment dans les pays d'élevage ou en raison des insecticides... 